SHORT REPORT

\title{
Cortical laminar necrosis related to prolonged focal status epilepticus
}

\author{
A Donaire, M Carreno, B Gómez, P Fossas, N Bargalló, R Agudo, M Falip, X Setoaín, T Boget, \\ T Raspall, V Obach, J Rumiá
}

J Neurol Neurosurg Psychiatry 2006;77:104-106. doi: 10.1136/jnnp.2004.058701

\begin{abstract}
Cortical laminar necrosis (CLN) is radiologically defined as high intensity cortical lesions on T1 weighted MRI images following a gyral distribution. Histopathologically, CLN is characterised by pannecrosis of the cortex involving neurones, glial cells, and blood vessels. It has been reported to be associated with hypoxia, metabolic disturbances, drugs, and infections. We present two patients who developed CLN and permanent neurological deficits after prolonged and repeated focal status epilepticus. The possible mechanisms leading to CLN in these patients are discussed, together with the implications of prompt and aggressive treatment in similar cases.
\end{abstract}

C ortical laminar necrosis (CLN) is a permanent brain injury radiologically characterised by high intensity cortical lesions on Tl weighted MRI images which follow the gyral anatomy of the cerebral cortex. ${ }^{1}$ In the few cases in which pathological studies have been performed, these images showed focal destruction of the cerebral cortex involving neurones, glial cells, and blood vessels without haemorrhage or calcification. ${ }^{1}$ We report two patients who developed CLN accompanied by persisting focal neurological deficits after prolonged focal status epilepticus (SE).

\section{CASE DESCRIPTIONS}

\section{Patient 1}

A 62 year old right handed man with history of acute bilateral otitis media was admitted after being found unconscious. An initial CT scan showed a brain abscess in the right temporal lobe. The patient developed left face clonic SE without impairment of consciousness 5 days after admission. The EEG showed periodic lateralised epileptiform discharges (PLEDs) over the right temporal region. A brain MRI showed the brain abscess as well as cortical high intensity lesions on T2 weighted images associated with mild subcortical oedema involving the right posterior temporal, parietal, and occipital regions (fig 1B, Bl). These lesions were interpreted as acute changes associated with SE. The patient was administered i.v. phenytoin and the seizures stopped 3 days later. Physical examination upon discharge showed no focal neurological deficits. However, 6 weeks later, while the phenytoin dose was being reduced, the patient developed recurrent left hand and face clonic SE. The patient was afebrile. Physical examination showed continuous clonic movement of the left face and fingers of the left hand as well as mild left arm paresis and a moderate to severe hypoaesthesia in the left arm. A left homonymous hemianopia and sensory extinction over the left hemibody were also observed. A new brain MRI showed high intensity cortical lesions on T2 weighted images in the right posterior temporal, parietal, and occipital regions similar to those observed on the MRI performed during the first SE (fig 1B, B2). Ictal SPECT showed hyperperfusion over the right opercular region and the entire right posterior quadrant (fig lA). The patient was administered i.v. phenytoin and a high dose (1500 mg) of oral levetiracetam. Seizures were not completely controlled until 4 days later. Final brain MRI performed 3 weeks after the second SE showed high intensity cortical lesions on $\mathrm{Tl}$ weighted images with a laminar pattern following the gyral anatomy in the right posterior temporal, parietal, and occipital regions characteristic of CLN (fig 1B, B3-4). Upon discharge the patient showed hypoaesthesia for all sensory modalities over the left arm, as well as visual and sensory extinction.

These neurological deficits still persist 5 months later. Follow up MRI has shown persistence of CLN over the posterior quadrant of the right hemisphere.

\section{Patient 2}

A 43 year old right handed man with a past medical history significant for epilepsy symptomatic of a left parieto-occipital subcortical arteriovenous malformation (AVM) was admitted to hospital because of right arm clonic SE. Neurological examination showed disorientation with respect to time and place, poor language production, and intermittent right arm clonic movements. An EEG showed PLEDs over the left parieto-occipital region.

An urgent CT scan showed no signs of cerebral haemorrhage. Blood pressure, blood cell counts, and blood biochemistry were normal. The patient was given i.v. valproic acid and phenytoin. In spite of therapeutic blood levels of both antiepileptic drugs (AEDs), focal motor seizures did not completely cease until 5 days later. A brain MRI performed during SE showed cortical hyperintensity on DP/T2 weighted images in the left posterior temporal, parietal, and occipital regions (fig $1 \mathrm{C}, \mathrm{Cl}-2$ ) in addition to the AVM. Physical examination after the SE showed a right homonymous hemianopia and mild receptive aphasia which were both fully resolved in 5 days and were interpreted as a Todd's-like phenomenon. However, 2 weeks later, the patient again experienced focal motor SE in spite of therapeutic AED levels and no obvious precipitating factor.

Treatment with phenytoin and valproic acid was maximised and oral levetiracetam was added, but seizures were not controlled until 8 days later. A brain MRI performed 1 week after the seizures showed prominent cortical Tl hyperintensity over the left posterior temporal, parietal, and occipital regions, indicative of CLN (fig 1C, C3-4). By then, physical examination showed a complete right homonymous hemianopia, a mild right hemiparesis, and severe receptive

Abbreviations: AED, antiepileptic drug; AVM, arteriovenous malformation; CLN, cortical laminar necrosis; CVR, cerebrovascular reserve; NO, nitric oxide; PLEDs, periodic lateralised epileptiform discharges; SE, status epilepticus 

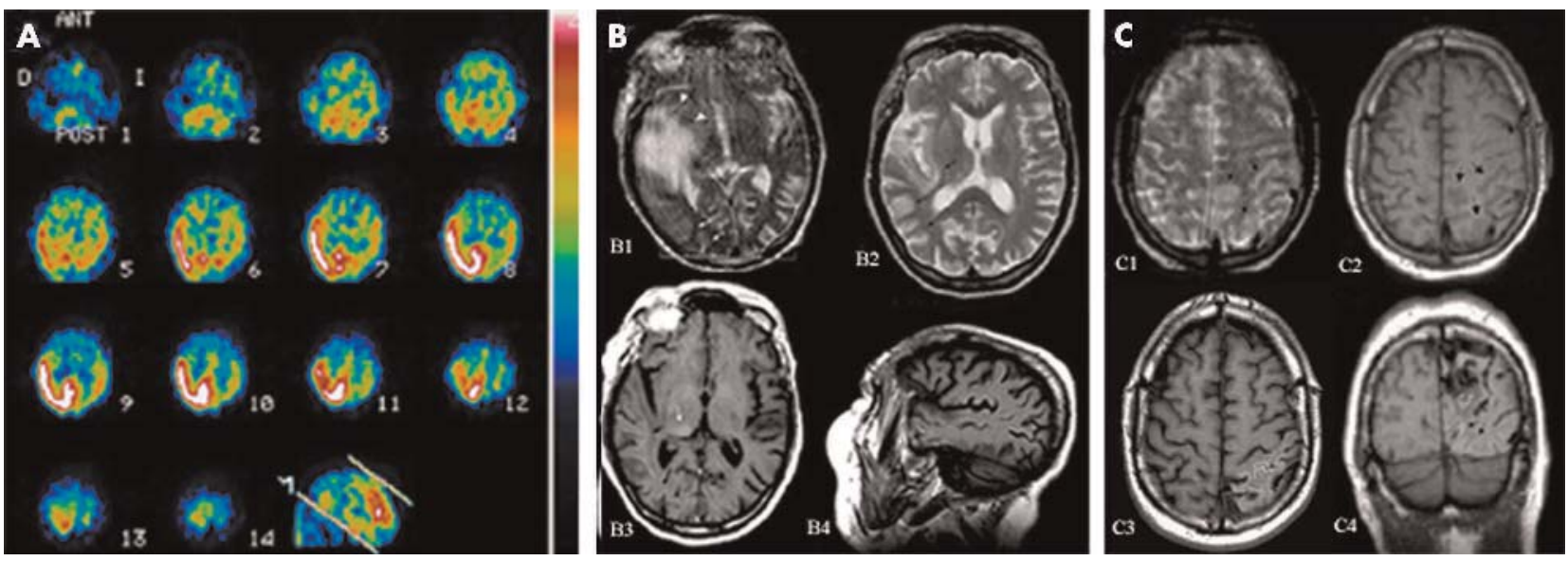

Figure 1 (A) Ictal 99mHMPAO SPECT demonstrating right temporo-parieto-occipital hyperperfusion. (B1-2) Axial T2 weighted images showing cortical increased signal intensity of the right temporo-parieto-occipital regions compatible with transient radiological changes associated with SE. B1 corresponds to the first SE. Notice the extensive cortical and white matter oedema associated with the brain abscess compared to the exclusively cortical oedema related to radiological changes associated with SE. The patient recovered after the first SE without significant neurological deficits. B2 corresponds to the second SE. At this time the oedema was mostly cortical and there were no signs of acute infection in the temporal region. (B3-4) Follow-up MRI, 3 weeks after the second SE. Axial and sagittal T1 weighted images show a high intensity cortical laminar lesion following a gyral distribution associated with volume loss in the right posterior temporal, parietal, and occipital regions, indicative of CLN. Notice also a hyperintense lesion on T1 weighted images located at the posterolateral portion of the ipilateral thalamus (C2). (C1-2). Axial T1/T2 weighted images showing cortical increased signal intensity on T2 weighted images $(\mathrm{C} 1)$ of the left parietal region and decreased signal intensity in the exact same location in $\mathrm{Tl}$ weighted images (C2) during the first SE. Notice the absence of hyperintense cortical lesions in the posterior quadrant during the first SE in C2. (C3-4) Follow up MRI, 1 week after the second SE. Axial and sagittal T1 weighted images show a high intensity cortical lesion following a gyral pattern associated with volume loss in the left posterior temporal, parietal, and occipital regions.

aphasia. The right hemiparesis resolved within approximately l week, but the right homonymous hemianopia and the receptive aphasia remained unchanged 7 months later. Follow up MRI at this time shows persisting CLN with the same distribution on the left posterior quadrant.

\section{DISCUSSION}

Transient radiological changes, such as hyperintense cortical lesions in T2, FLAIR, and diffusion weighted images, have been reported in patients with focal SE. ${ }^{2-4}$ Our two patients displayed these acute changes during focal motor SE. MRI changes during SE are reversible in most patients, but in some cases permanent brain damage has been reported. ${ }^{3-8}$ Our two patients displayed permanent brain imaging abnormalities consistent with CLN after prolonged focal SE. Radiologically, CLN is characterised by high intensity cortical lesions on Tl weighted and FLAIR images following a gyral distribution, associated with volume loss over the underlying cortex. CLN has been described associated with hypoxia, metabolic disturbances, drugs, infections, ${ }^{9}$ and SE. Only three cases of CLN associated with SE have been previously reported, and all had severe hypoglycaemia or hypoxia during the episode. ${ }^{6}{ }^{10}$ In contrast, our two patients did not show any metabolic disturbances or significant decreases in blood pressure during the episodes of focal SE. The hypothesis that the necrosis observed in these patients is primarily a consequence of repeated seizures is further supported by the fact that CLN is seen in the same areas as those displaying acute cortical oedema and hyperperfusion (as shown by ictal SPECT in patient number 1) during the acute phase of SE. Furthermore, these hyperintense lesions did not involve separate vascular territories and occurred at a distance from the structural lesions. The location of CLN was also concordant with the location of continuous epileptic activity shown by surface EEG during SE.

It can be speculated that CLN in the first patient was due to cerebritis in the context of a brain infection; however, the absence of focal neurological deficits during and after the first episode of SE (when the abscess was active) and the appearance of focal neurological deficits after the second $\mathrm{SE}$, when the infectious process was inactive, makes this hypothesis very unlikely. In the second patient, a haemodynamic ischaemic event ("steal phenomenon") related to the AVM as well as a venous infarction in the context of hyperperfusion secondary to SE in a vascular bed with impaired vasoreactivity, can be reasonably ruled out as the main pathogenic mechanism. As there are no signal changes in the white matter under the involved cortex reflecting vasogenic oedema or blood-brain barrier rupture and also because the CLN extends far beyond the AVM and crosses vascular territories.

In our patients, CLN correlated clinically with severe focal neurological deficits (cortical sensory disturbance in patient number 1 , hemiparesis, aphasia, and hemianopia in patient number 2) which have remained unchanged over time.

Therefore, prolonged focal SE can cause permanent brain damage; this is the first time that CLN has been described as being associated with focal SE in the absence of other metabolic or toxic factors. Many mechanisms can lead to permanent brain damage as a result of SE. Status epilepticus causes a large increase in cerebral metabolic demand for glucose and oxygen and there is, therefore, a compensatory increase in cerebral blood flow. If blood flow is not sufficient, adenosine triphosphate depletion and lactate accumulation occur, leading to hypermetabolic neural necrosis. ${ }^{11}$ Experimental animal studies have shown that correlation between regions with excessive metabolism and the distribution and extent of seizure induced neuronal damage is quite good. ${ }^{12}$ There are also excitotoxic mechanisms mediated by both NMDA and non-NMDA receptors resulting in $\mathrm{Ca}^{2+}$ entry and release of $\mathrm{Ca}^{2+}$ from intracellular stores which plays a major role in neuronal injury from SE. ${ }^{611}$

The location of the lesions, in the posterior quadrant of the cerebral hemispheres in our patients, may be related to the poverty of sympathetic innervation of the vessels arising from the basilar artery; it is known that this innervation plays a fundamental role in cerebrovascular reactivity that leads to coupling between cortical activity and cerebral blood flow. ${ }^{13}{ }^{14}$ 
Furthermore, experimental animal studies $^{15}$ examining vasoreactivity in response to nitric oxide (NO) assessed by cerebral angiography, showed that the vascular response to NO was greater in the posterior than the anterior circulation. Taking into account the fact that basal release of NO contributes to the maintenance of the resting vascular tone, it could be hypothesised that the posterior circulation is more dilated under baseline conditions and therefore has less capacity for further vasodilatation when needed. This may lead to a reduced cerebrovascular reserve (CVR) and uncoupling between the cortical metabolic demand and the cerebral blood flow in those situations in which high metabolic demands are required, such as focal SE. However, this needs to be confirmed, as other human studies assessing CVR and $\mathrm{CO}_{2}$ reactivity using transcranial Doppler ultrasound have not found significant differences in vasoreactivity between the vertebro-basilar and carotid systems. ${ }^{16}{ }^{17}$

In conclusion, most of the radiological cortical signal changes associated with focal SE seem to be reversible. However, a group of patients may display permanent brain damage after SE, with radiological changes suggestive of CLN and accompanying focal neurological deficits. It is possible that patients who display hyperintense cortical signal changes during the acute phase of SE are more at risk of developing permanent brain damage, but larger series of patients with follow up MRI studies are needed to answer this question. It is also unclear if certain cortical areas are more susceptible to seizure induced permanent damage and cortical necrosis. Interestingly enough, our patients had focal SE involving the posterior quadrant of the hemisphere. The possibility of permanent brain damage after focal SE, even without alteration of consciousness, should encourage prompt and aggressive treatment of recurrent seizures.

\footnotetext{
Authors' affiliations

A Donaire, M Carreno, B Gómez, N Bargalló, R Agudo, M Falip,

X Setoaín, T Boget, T Raspall, V Obach, J Rumiá, Hospital Clínic de Barcelona, Barcelona, Spain

P Fossas, Hospital General Mataró, Barcelona, Spain

Competing interests: none declared

The patients detailed in this report gave their informed consent for their details to be published
}

Correspondence to: Dr Mar Carreno, Epilepsy Unit, Hospital Clínic de Barcelona, Villarroel 170, 08036, Barcelona, Spain; mcarreno@clinic. ub.es

Received 14 November 2004

Revised version received 29 April 2005

Accepted 4 May 2005

\section{REFERENCES}

1 Komiyama M, Nakajima $H$, Nishikawa M, et al. Serial MR observation of cortical laminar necrosis caused by brain infarction. Neuroradiology 1998;40:771-7.

2 Kramer RE, Luders H, Lesser RP, et al. Transient focal abnormalities of neuroimaging studies during focal status epilepticus. Epilepsia 1987;28:528-32.

3 Meierkord H, Wieshmann U, Niehaus L, et al. Structural consequences of status epilepticus demonstrated with serial magnetic resonance imaging. Acta Neurol Scand 1997;96:127-32.

4 Sammaritano M, Andermann F, Melanson D, et al. Prolonged focal cerebral edema associated with partial status epilepticus. Epilepsia 1985;26:334-9.

5 Chan S, Chin SS, Kartha K, et al. Reversible signal abnormalities in the hippocampus and neocortex after prolonged seizures. AJNR Am J Neuroradiol 1996;17:1725-31.

6 Kim JA, Chung Jl, Yoon PH, et al. Transient MR signal changes in patients with generalized tonicoclonic seizure or status epilepticus: periictal diffusionweighted imaging. AJNR Am J Neuroradiol 2001;22:1149-60.

7 Lansberg MG, O'Brien MW, Norbash AM, et al. MRI abnormalities associated with partial status epilepticus. Neurology 1999;52:1021-7.

8 Nohria V, Lee N, Tien RD, et al. Magnetic resonance imaging evidence of hippocampal sclerosis in progression: a case report. Epilepsia 1994;35:1332-6.

9 Bargallo N, Burrel M, Berenguer J, et al. Cortical laminar necrosis caused by immunosuppressive therapy and chemotherapy. AJNR Am J Neuroradiol 2000;21:479-84.

10 Christiaens FJ, Mewasingh LD, Christophe C, et al. Unilateral cortical necrosis following status epilepticus with hypoglycemia. Brain Dev 2003;25:107-12.

11 Wasterline CJ, Fujikawa DG, Penix L, et al. Pathophysiological mechanisms of brain damage from status epilepticus. Epilepsia 1993;34:37-53.

12 da Silva Frenandes MJ, Dubé C, Boyet S, et al. Correlation between hypermetabolism and neural damage during status epilepticus induced by lithium and pilocarpine in immature and adult rats. J Cereb Blood Flow Metab 1999;19(2): 195-209.

13 Paulson OB. Blood-brain barrier, brain metabolism and cerebral blood flow. Eur Neuropsychopharmacol 2002;12:495-501.

14 Rosengarten B, Huwendiek O, Kaps M. Neurovascular coupling in terms of control system: validation of a second-order linear system model. Ultrasound Med Biol 2001;27:631-5.

15 Kajita Y, Takayasu M, Suzuki Y, et al. Regional differences in cerebral vasomotor control by nitric oxide. Brain Res Bull 1995;38:365-9.

16 Barret KM, Ackerman RH, Gahn G, et al. Basilar and middle cerebral artery reserve. Stroke 2001;32:2793-6.

17 Wan Park C, Sturzenegger M, Douville CM, et al. Autoregulatory response and CO2 reactivity of basilar artery. Stroke 2003;34:34-9. 